\title{
Analysis and design of an interdigital cantilever as a displacement sensor
}

\author{
G. G. Yaralioglua) and A. Atalar \\ Electrical and Electronics Engineering Department, Bilkent University, Ankara 06533, Turkey
}

S. R. Manalis and C. F. Quate

Ginzton Laboratory, Stanford University, Stanford, California 94305

(Received 23 January 1998; accepted for publication 19 March 1998)

\begin{abstract}
The interdigital (ID) cantilever with two sets of interleaving fingers is an alternative to the conventional cantilever used in the atomic force microscope (AFM). In this paper we present a detailed analysis of the interdigital cantilever and its use as a sensor for the AFM. In this study, we combine finite element analysis with diffraction theory to simulate the mechanically induced optical response of the ID. This model is used to compare this system with the optical lever detector as used in conventional instruments by analyzing the ratio of signal to noise and overall performance. We find that optical detection of the cantilever motion with interdigital fingers has two advantages. When used in conjunction with arrays of cantilevers it is far easier to align. More importantly, it is immune to laser pointing noise and thermally excited mechanical vibrations and this improves the sensitivity as compared to the optical lever. (C) 1998 American Institute of Physics.
\end{abstract}

[S0021-8979(98)07112-6]

\section{INTRODUCTION}

The atomic force microscope (AFM), ${ }_{1}^{1}$ or scanning probe microscope, provides high resolution images of surfaces. It is based on sensing the interaction force between the surface and the cantilever tip. As the cantilever is scanned across the surface, it bends in proportion to the force between the tip and the sample. The deflection of the cantilever is measured with various systems. The most common sensors include interferometry, ${ }^{2,3}$ the optical lever, ${ }^{4,5}$ and a piezoresistive element ${ }^{6}$ used to sense the strain. The sensitivity with these methods is sufficient to resolve features on the atomic scale and indeed this fine sensitivity is a basic factor in the widespread acceptance of the AFM.

A highly sensitive technique for measuring the deflection of a cantilever is the interferometer. Rugar et $\mathrm{al}^{3}{ }^{3}$ developed a deflection sensor based on the interference of light between the cleaved end of an optical fiber and the backside of a cantilever. By accurately positioning the fiber above the cantilever to form a tightly spaced interference cavity of less than $4 \mu \mathrm{m}$, it is possible to achieve a vertical resolution on the order of $0.01 \AA$.

One of the most common techniques used to measure the deflection of a cantilever is the optical lever. In this system, a laser beam is reflected off the backside of the cantilever and directed into a split photodiode. The position of the reflected beam, and hence the cantilever deflection, is determined by subtracting the photodiode outputs. Unlike the interferometer, the optical lever does not require the positioning of components directly above the cantilever. It is this simplicity that has made the optical lever more popular than the interferometer. However, the resolution is typically limited to roughly $0.1 \AA$.

\footnotetext{
a)Electronic mail: goksenin@ bilkent.edu.tr
}

The deflection of a cantilever can also be determined with an integrated piezoresistive strain gauge. Since silicon is a piezoresistive material, it can be used to microfabricate cantilevers that change resistance when stressed. Developed by Tortonese, ${ }^{6}$ the piezoresistive cantilever is capable of 0.1 $\AA$ resolution in a $10 \mathrm{~Hz}-1 \mathrm{kHz}$ bandwidth. The main advantage of using a piezoresistor to measure cantilever deflection is that alignment is not required. In the case of the optical lever, there are typically two alignment steps that require physical positioning: first, a laser must be aligned to the end of the cantilever and second, a split-photodiode must be aligned to the laser beam that reflects off the cantilever. When using the piezoresistor, it is only necessary to balance the resistor bridge by changing the resistance of one of the elements. For low temperature or ultrahigh vacuum (UHV) applications where physical alignment is difficult, the piezoresistor is a simple alternative. The piezoresistor is also a useful technique for measuring the deflection of cantilever arrays. ${ }^{7}$

The advances in silicon micro-machining techniques permit us to fabricate cantilevers with intricate designs and small dimensions. Our new interferometric detection method, as introduced earlier, ${ }^{8}$ is based on a cantilever shaped to form an interdigital optical diffraction grating. The interdigital grating is composed of two sets of fingers. One set contains the tip which follows the contour of the sample. The other set is rigidly connected to the cantilever support and remains stationary during scanning. When the fingers are illuminated, the optical beams reflected from fingers produce a diffraction pattern composed of many orders. The intensities of each order depend on the amount of cantilever deflection. In this way the cantilever deflection is determined by a simple measurement of optical intensity and this gives us the simplification that is needed to adapt this system to cantilever arrays. 


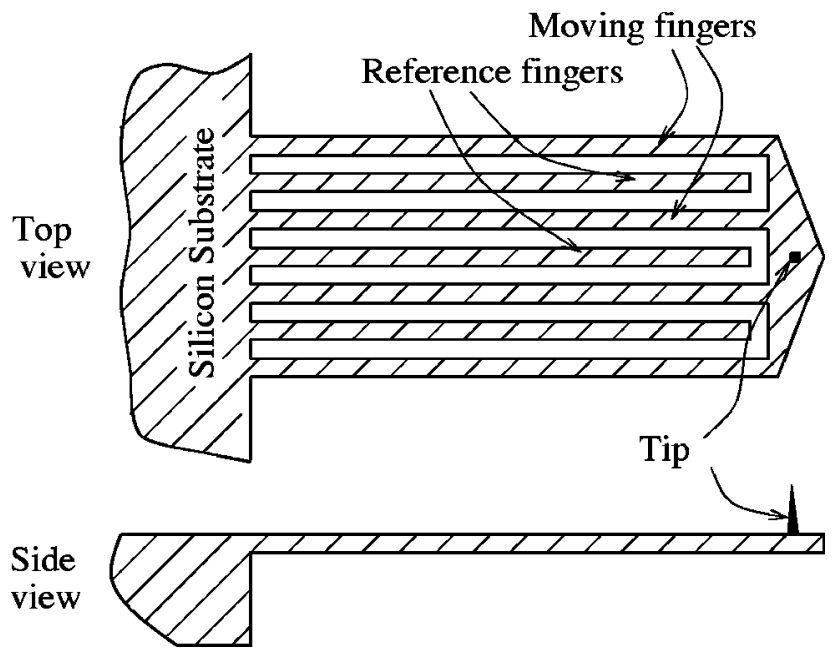

FIG. 1. Geometry of the first kind interdigital cantilever.

In the following sections we present a detailed analysis of the operation of the interdigital cantilever. We will first introduce the geometry and the associated process of fabrication and then formulate the theory underlying the operation of the phase gratings with the responses curves and confirming experimental results. The noise performances of the interdigital (ID) cantilever will be compared to the optical lever detection method. We will conclude with a discussion of the overall advantages of the ID cantilever.

\section{GEOMETRY OF INTERDIGITAL CANTILEVER}

There are two ways of implementing phase gratings on cantilevers. Figure 1 shows the first kind of cantilever where the fingers are directed along the direction of the cantilever axis. In the second kind the fingers are perpendicular to cantilever axis (Fig. 2). There is little difference between the two geometries except for the axis of diffraction pattern which is perpendicular to the cantilever for the first kind and parallel to the cantilever axis for the second kind. The geometry of the first kind is more simple in some ways, but it is not suitable for arrays since the higher order diffraction patterns from neighboring cantilevers interfere with each other.

The typical ID cantilever is several micrometers thick, several hundred micrometers in length and $100 \mu \mathrm{m}$ in width.

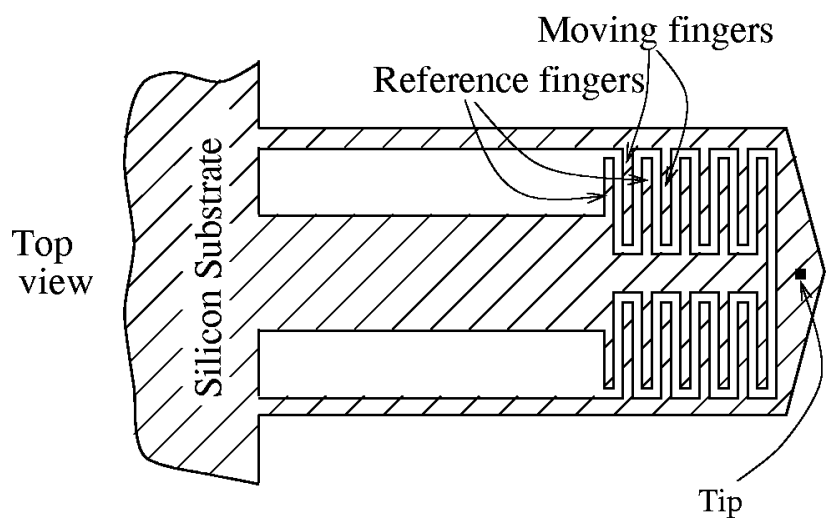

FIG. 2. Geometry of the second kind interdigital cantilever.

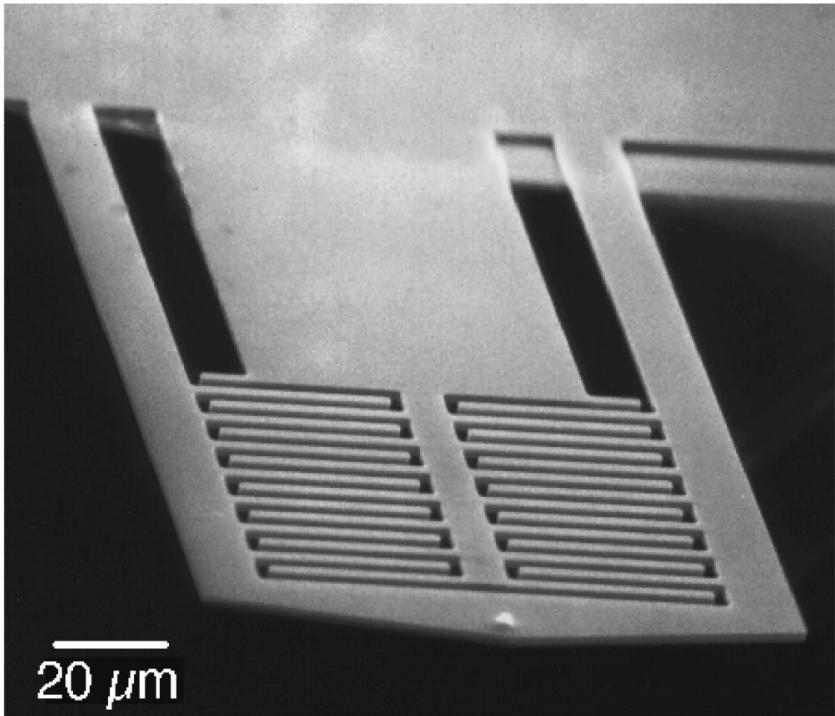

FIG. 3. SEM image of an interdigital cantilever. The length of the cantilever is $215 \mu \mathrm{m}$. The length and the width of the fingers are 30 and $3 \mu \mathrm{m}$, respectively. There are seven finger pairs $(N=7)$. The thickness of the structure is $2.5 \mu \mathrm{m}$.

A sharp tip perpendicular to this surface is formed at the end. The cantilever is fabricated from silicon with the standard techniques of micro-machining. Alternatively, silicon nitride can be used in place of silicon and the surface of the fingers is coated with an optically reflecting material such as aluminum or gold. Fabrication of the interdigital cantilever is a three mask process that begins by growing $1 \mu \mathrm{m}$ of thermal oxide on a $\langle 100\rangle$ silicon-on-insulator (SOI) wafer where the uppermost layer is undoped epitaxial silicon $10 \mu \mathrm{m}$ in thickness. Tip masks are patterned into the oxide with 6:1 HF, undercut into the epitaxial silicon with a plasma etch, and sharpened by a wet oxidation at $950{ }^{\circ} \mathrm{C}$ for $2 \mathrm{~h}$. The cantilever and the interdigitated fingers are defined in a plasma etch. The top surface is then passivated with polyimide and the bulk silicon is etched with ethylene diamine pyrocathecol (EDP) using the middle oxide as an etch stop. Cantilevers are released by etching the middle oxide in 6:1 $\mathrm{HF}$ and removing the polyimide in an oxygen plasma.

A scanning electron micrograph of the ID cantilever of the second kind is shown in Fig. 3. The tip is visible on the triangular piece at the end of the cantilever. One set of fingers is connected to the outer portion of the cantilever which moves when a force is applied to the tip. The second set of fingers is connected to the inner portion which remains fixed.

We have used a general purpose finite-element package, ANSYS version $5.2^{9}$ to study the shape of the modes and the associated resonances. A four-node elastic shell element (SHELL63) was used to construct the finite element model (FEM) model. This resonance is important since the high frequency limit of the imaging bandwidth is set by the first resonance peak of the cantilever. The calculated and experimentally measured resonance frequency of our cantilever is around $46 \mathrm{kHz}$. This is the first longitudinal resonance of the outer portion of the cantilever. At this frequency, the triangular part of the cantilever moves up and down. The second 


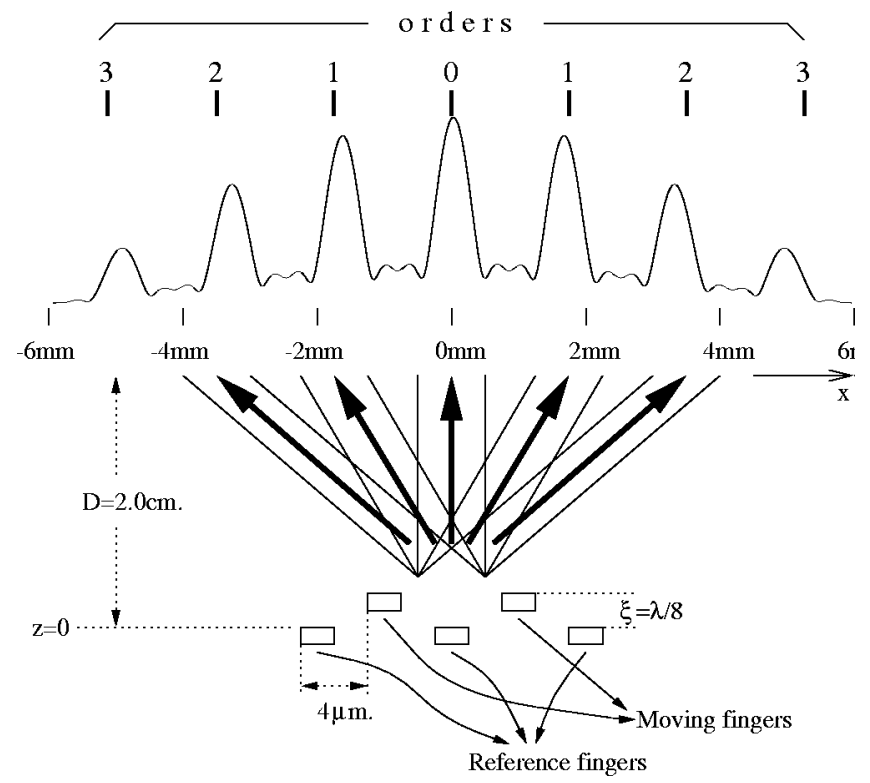

FIG. 4. Cross-sectional view of the grating. The width of the fingers are 2 $\mu \mathrm{m}$. Spatial frequency of the grating is $f_{g}=5 \times 10^{5} \mathrm{~m}^{-1}$.

resonance frequency is the first longitudinal resonance of the inner part. The third mode corresponds to a torsional mode where the cantilever rotates around the axis of the cantilever. The individual fingers resonate at a frequency above $3 \mathrm{MHz}$.

\section{THEORY}

The geometry of the interdigital cantilever forms a phase sensitive optical diffraction grating. This grating reflects the incident coherent optical beam into several orders with an intensity that depends on the relative displacement between the two sets of fingers. Figure 4 shows the cross section of the grating and the profile of the optical diffraction pattern. In the equilibrium position, $\xi=0$, where $\xi$ represents the relative deflection of moving fingers with respect to reference fingers, the intensities of the even-numbered orders are maximum [Fig. 5(a)]. The spatial separation of the second order component from the central component (the zeroth order) is $\lambda D f_{g}$, where $f_{g}$ is the spatial frequency of the grating, $D$ is the observation distance and $\lambda$ is the wavelength of the incident beam. When the moving fingers are displaced by $\lambda / 4$, the central beam vanishes and the energy is divided between the two first order components and other odd numbered components [Fig. 5(b)]. Figure 5 was calculated from fingers of infinite length. The diffraction pattern profile is calculated by taking the one-dimensional Fourier transform of the grating. If we assume the amplitude of the incident beam varies as $\cos (\omega t+k z)$, where $k$ is the wave number, we can calculate the intensity of the zeroth order component as a function of cantilever deflection. At $z=0$ the amplitudes of the beam reflected from the two sets of fingers are $\cos (\omega t)$ and $\cos (\omega t+2 k \xi)$, respectively. If we add these two cosine terms, we find that the intensity of the zeroth order component, $I_{0}$, is proportional to

$$
I_{0} \propto \cos ^{2} \theta,
$$

where

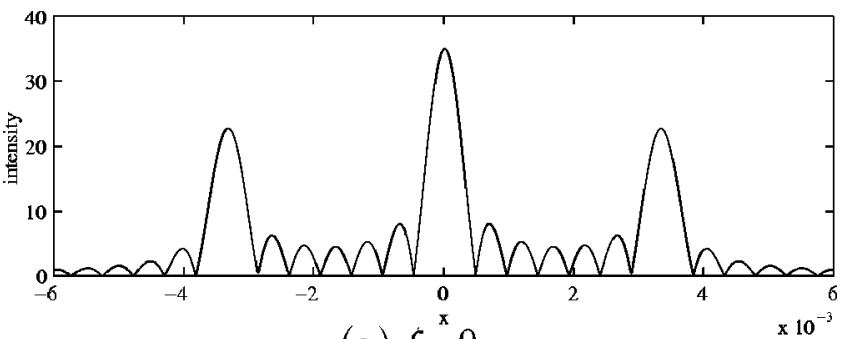

(a) $\xi \stackrel{x}{=} 0$

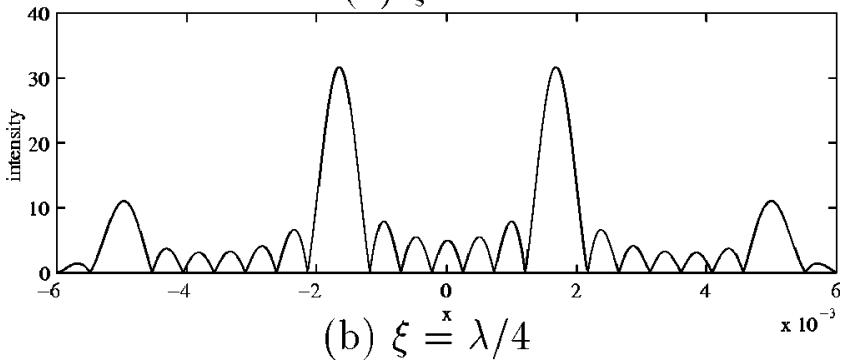

FIG. 5. Field intensity at $D=2 \mathrm{~cm}$. Fingers are assumed to be infinitely long.

$$
\theta=\frac{2 \pi}{\lambda} \xi
$$

The reflected beams from moving fingers and reference fingers add constructively when $\xi=0, \lambda / 2, \lambda, 3 \lambda / 2 \ldots$. Similarly, the intensity of the first order component, $I_{1}$, is proportional to

$$
I_{1} \propto \sin ^{2} \theta .
$$

Again, reflected beams from moving fingers and reference fingers add constructively when $\xi=\lambda / 4,3 \lambda / 4,5 \lambda / 4 \ldots$.

The phase difference between incident and reflected beam is $2 k \xi$, when we assume that the incident beam is normal to the cantilever plane, i.e., the incidence angle is $0^{\circ}$. Experimentally, it is difficult to illuminate the cantilever with this angle of incidence and measure the diffraction pattern at the same time since there is usually a small incidence angle, $\gamma$. If the effect of the incidence angle is considered, in the above formulas, $\xi$ should be replaced by $\xi \cos \gamma$. We note that we maximize the sensitivity when the incidence angle is kept as small as possible.

Another issue that must be considered when designing interdigital cantilevers is the spatial separation of the orders. If the orders are not well separated, they interfere with each other and this reduces the sensitivity. The beam width for an order at the observation plane is proportional to $\lambda D f_{g} / N$, where $N$ is the number of finger pairs and $N / f_{g}$ is the length of the grating. The ratio of the spatial separation between successive orders to the beam width ${ }^{10}$ can be considered as a figure of merit and it is given by

$$
\frac{f_{g} \lambda D / 2}{\lambda D f_{g} / N}=N / 2 \text {. }
$$

This ratio is proportional to the number of fingers, but it is independent of observation distance $D$. We conclude that if $N$ is greater than 4 , the orders are well separated. 


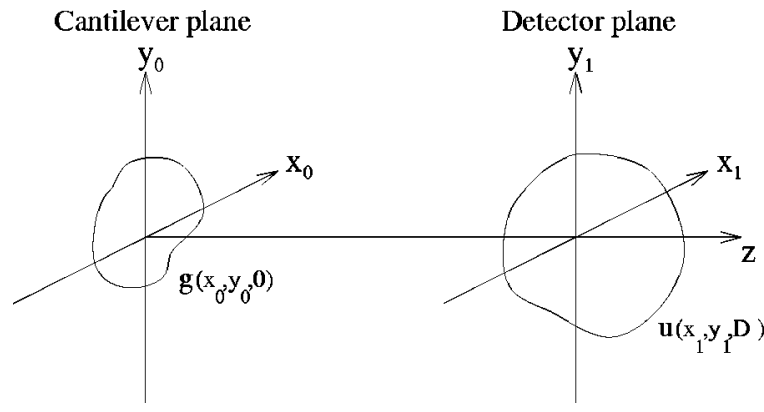

FIG. 6. Coordinate system.

\section{SIMULATIONS AND EXPERIMENTS}

In order to simulate the performance of the interdigital cantilever, the diffraction pattern above the interdigitated fingers has to be determined. The diffraction pattern from an arbitrary source distribution can be found by using the wellknown diffraction integral ${ }^{11}$ which can be difficult to calculate. However, Fresnel approximations for near field calculations are very accurate and computationally less complex. The Fresnel formula for calculating field amplitude due to an arbitrary source distribution is given by the Fourier transform of the source distribution multiplied by a constant phase surface. With the notation defined in Fig. 6, the resulting field, $u\left(x_{1}, y_{1}\right)$, due to an arbitrary source distribution, $g\left(x_{0}, y_{0}\right)$, is given by

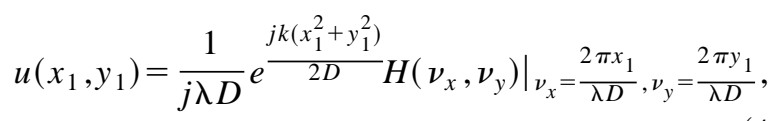

where

$$
H\left(\nu_{x}, \nu_{y}\right)=F\left\{g\left(x_{0}, y_{0}\right) e^{\frac{j k\left(x_{0}^{2}+y_{0}^{2}\right)}{2 D}}\right\} .
$$

The light intensity at the $z=D$ plane is proportional to $\left|u\left(x_{1}, y_{1}\right)\right|^{2}$. Hence, the intensity is

$$
I\left(x_{1}, y_{1}\right)=\left.\frac{1}{(D \lambda)^{2}}\left|H\left(\nu_{x}, \nu_{y}\right)\right|_{\nu_{x}=\frac{2 \pi x_{1}}{\lambda D}, \nu_{y}=\frac{2 \pi y_{1}}{\lambda D}}\right|^{2} \text {. }
$$

The intensity is calculated from the two-dimensional fast Fourier transform. In Fig. 7(a), we show the intensity distribution at the cantilever plane, $z=0$. The displacement $\xi$ is a function of $x_{0}$ and $y_{0}$ and it is denoted by $\xi\left(x_{0}, y_{0}\right)$. The amplitudes of the moving parts are multiplied by a phase term, $\exp \left[4 \pi j \xi\left(x_{0}, y_{0}\right) / \lambda\right]$, which denotes the additional twoway phase difference due to the cantilever deflection, $\xi\left(x_{0}, y_{0}\right)$. When a force is applied to the tip, the deflection of the cantilever varies along the length of the cantilever. It is zero at the point where the cantilever is connected to the silicon substrate and maximum at the tip. The cantilever deflection, $\xi\left(x_{0}, y_{0}\right)$, is calculated as a function of $x_{0}$ and $y_{0}$ by using ANSYS. Figure 8 shows the calculated displacement distribution of the cantilever when the tip is deflected by 210 $\mathrm{nm}$ with a force of $23 \mathrm{nN}$ on the tip. The calculated spring constant is $1.1 \mathrm{Nt} / \mathrm{m}$.

The function $g\left(x_{0}, y_{0}\right)$ is obtained by weighing the cantilever pattern by a Gaussian beam, $\exp \left[-\left(x_{0}^{2}+y_{0}^{2}\right) / \sigma^{2}\right]$, where

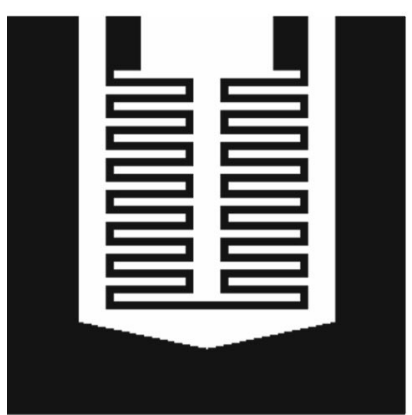

(a)

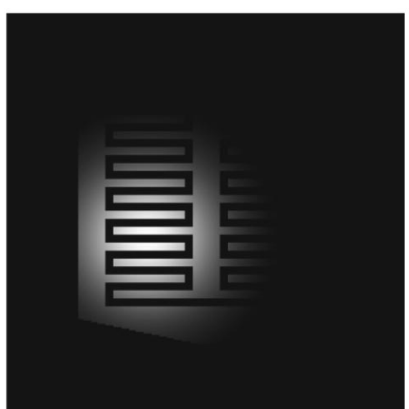

(b)
FIG. 7. (a) Cantilever pattern. (b) $g\left(x_{0}, y_{0}\right)$.

$\sigma=3.6 \mu \mathrm{m}$ [Fig. 7(b)]. Once $g\left(x_{0}, y_{0}\right)$ is determined, the intensity pattern at the desired $z=D$ plane is found by applying Eq. (4.3). In Fig. 9, we show the calculated diffraction pattern for various cantilever deflections.

With no deflection $\left[\xi\left(x_{0}, y_{0}\right)=0\right]$, the intensities of the even-numbered orders are maximum. For $D=4 \mathrm{~cm}$, the spatial separation of the second side order from the central component is $4.46 \mathrm{~mm}$ as calculated from $\lambda D f_{g}$. This value is consistent with Fig. 9. When the cantilever is deflected at $210 \mathrm{~nm}$, intensities of the odd-numbered components reach their maximum values. The distance between the first order component and the zeroth order component is around 2.2 $\mathrm{mm}$, which is nearly $\lambda D f_{g} / 2$.

The cantilever deflection can be determined by measuring the intensity of the zeroth order component, the first order component or the difference between the two. This is easily done by placing a photodetector at the proper position. Figure 10 shows the calculated detector output voltages versus cantilever deflection. For the detector output, we integrate over the area corresponding to the size of the photodetector. The period of the curve is slightly larger than the

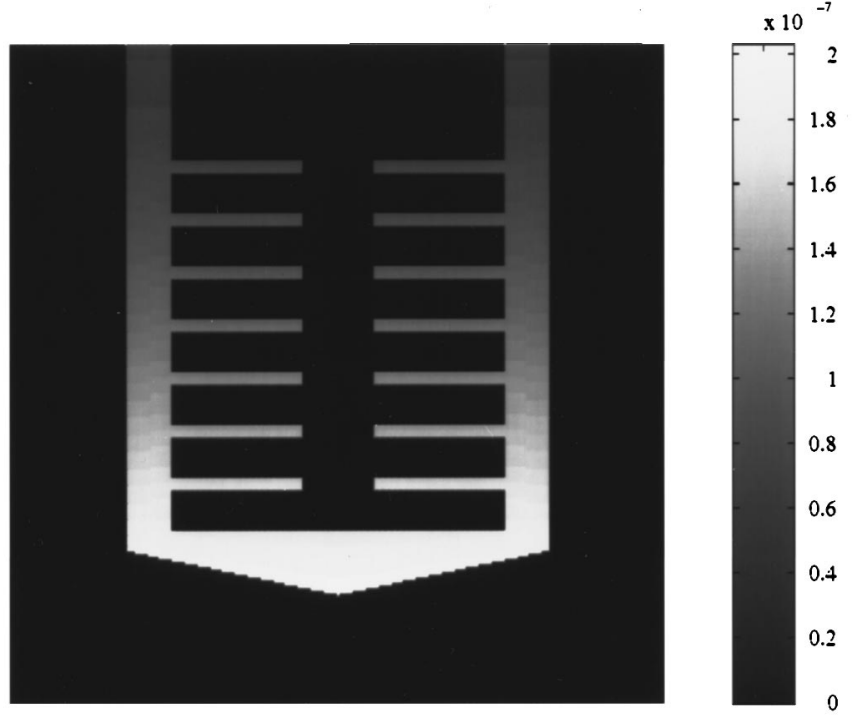

FIG. 8. Displacement of the moving fingers. (Young modulus, $E=130 \mathrm{~Pa}$, density, $\rho=2.332 \mathrm{~g} / \mathrm{cm}^{3}$, Poisson ratio, $\sigma=0.278$ ). 


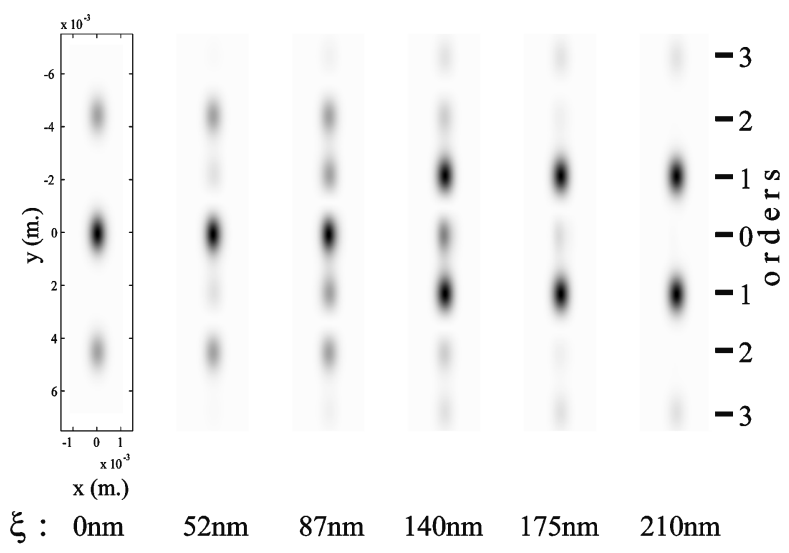

FIG. 9. Calculated diffraction pattern $4 \mathrm{~cm}$ above the interdigital cantilever for various deflections. The width and the spacing of the fingers are $3 \mu \mathrm{m}$ $\left(f_{g}=0.167 \times 10^{6} \mathrm{~m}^{-1}\right) . \xi$ shows the deflection at the tip position.

expected value $\lambda / 2$. This is due to the fact that the actual average displacement of the interdigital fingers is less than the displacement of the tip.

Figure 11 shows an experimental and calculated response curve. In the experiment, the interdigital cantilever of the second kind is illuminated by a laser beam with a spot size of $20 \mu \mathrm{m}(\sigma=3.6 \mu \mathrm{m})$ and the reflected diffraction pattern is measured with a split photodiode. The photodiode is placed so that the zeroth order mode illuminates one side and the first order mode illuminates the other. There is good agreement between the experimental and calculated data.

\section{COMPARATIVE NOISE ANALYSIS}

The minimum detectable deflection (MDD) is defined by a signal to noise ratio equal to unity. The main sources of noise in the deflection detection systems are shot noise of the photodetector, thermal mechanical noise of the cantilever, laser intensity noise, laser phase noise, laser $1 / f$ noise, laser pointing noise, resistor Johnson noise, electronic noise of the detection electronics and mechanical vibrations of the overall

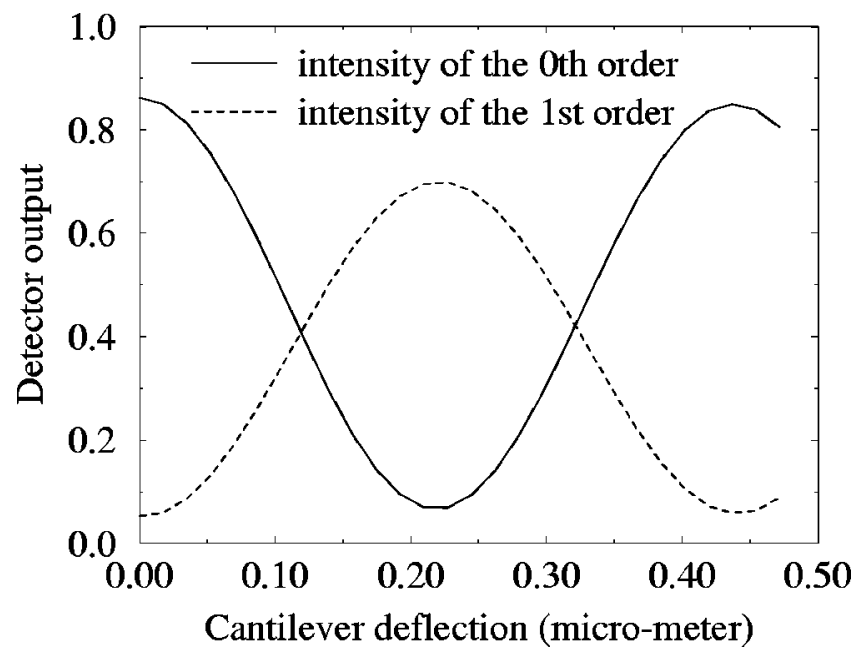

FIG. 10. Intensities of the zeroth and first order modes. Incidence angle, $\gamma$, is 0 . Detector is $200 \mu \mathrm{m} \times 200 \mu \mathrm{m}$.

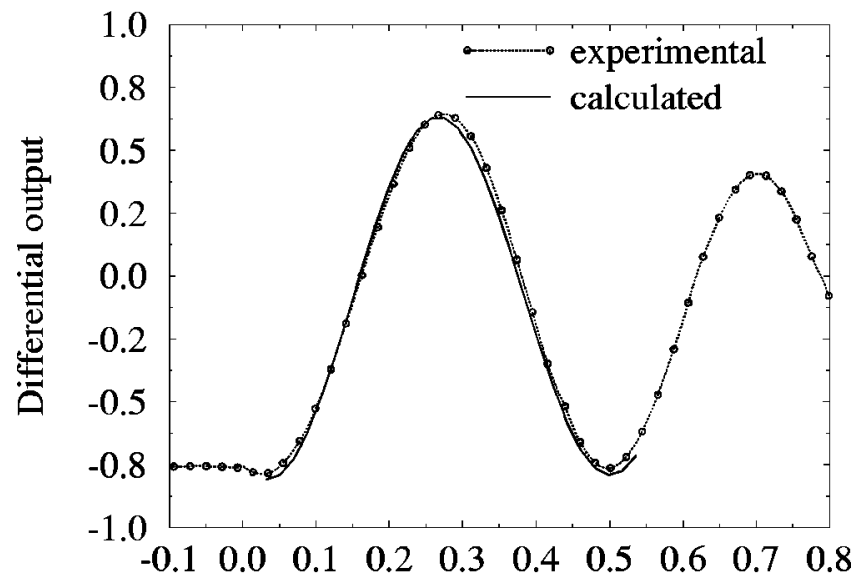

Cantilever deflection (micro-meter)

FIG. 11. Differential detector output. Experimental and calculated data. The length of the cantilever is $215 \mu \mathrm{m}$. Incidence angle is $20^{\circ}$.

system (Fig. 12). Figure 13 shows the equivalent noise circuit. The signal is denoted by a current source of value $i_{s}$, which is a function of the light intensity incident on the photodetector. For the definitions and the symbols of the noise currents see Appendix A. If the signal and noise powers from various sources are calculated from the circuit shown in Fig. 13, the signal to noise ratio (SNR) is found as

$$
\mathrm{SNR}=\frac{\left(\frac{d}{d \xi} i_{s}\right)^{2}}{\left\langle i_{\mathrm{sh}}^{2}\right\rangle+\left\langle i_{m}^{2}\right\rangle+\left\langle i_{\mathrm{ms}}^{2}\right\rangle+\left\langle i_{l}^{2}\right\rangle+\left\langle i_{e}^{2}\right\rangle},
$$

where

$$
\left\langle i_{l}^{2}\right\rangle=\left\langle i_{\text {int }}^{2}\right\rangle+\left\langle i_{\text {pha }}^{2}\right\rangle+\left\langle i_{p}^{2}\right\rangle+\left\langle i_{\text {ffl }}^{2}\right\rangle,
$$

and

$$
\left\langle i_{e}^{2}\right\rangle=\left\langle i_{n}^{2}\right\rangle+\frac{\left\langle e_{n}^{2}\right\rangle}{R^{2}}+\frac{\left\langle e_{R}^{2}\right\rangle}{R^{2}}+\left\langle i_{\mathrm{fe}}^{2}\right\rangle .
$$

Note that for the purposes of calculating the signal power, the derivative of the output current is used rather than the current itself. For the SNR calculation, the signal is defined as the change in the output current of the photodetector per unit displacement in the cantilever position. Our definition of

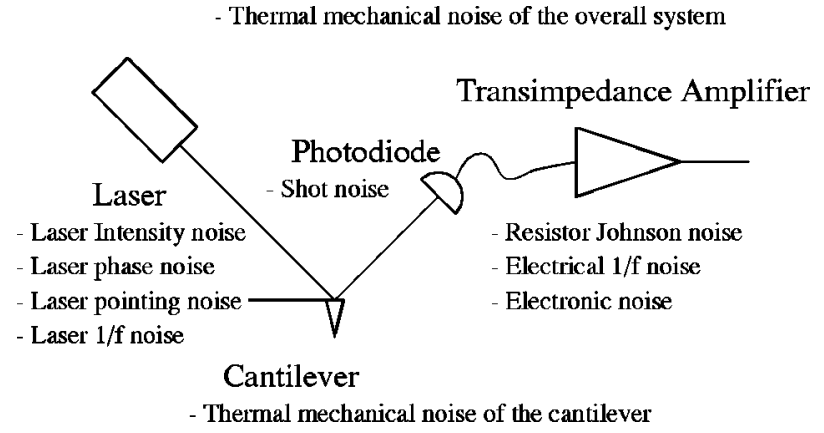

FIG. 12. Noise sources in a typical AFM system which uses optical detection methods. 


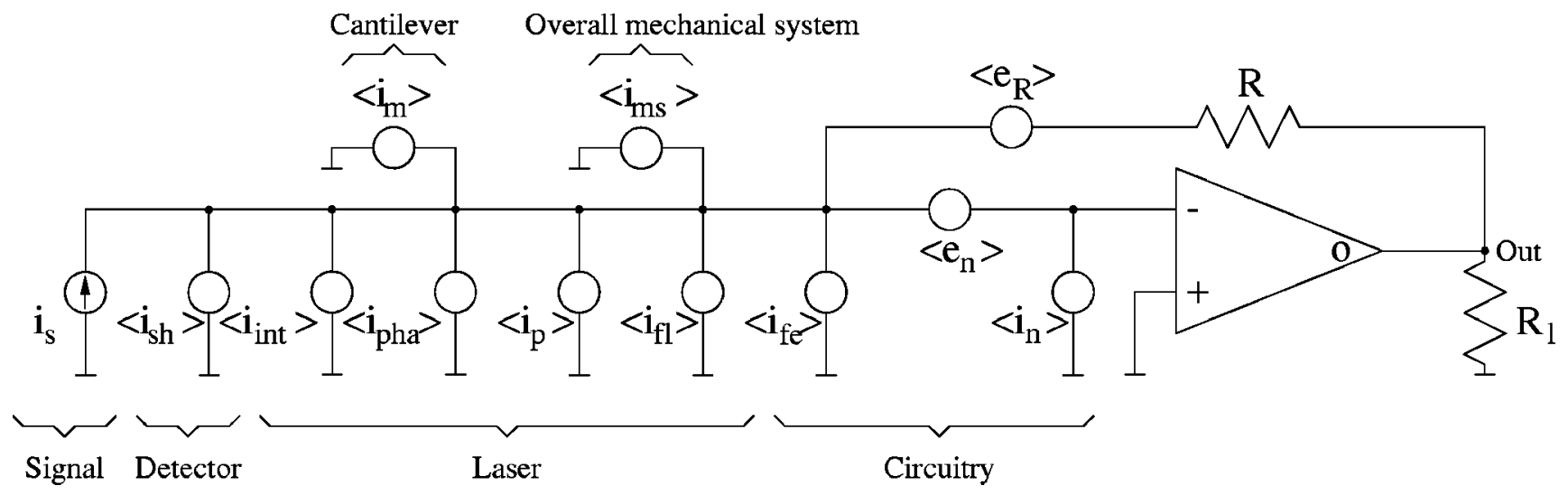

FIG. 13. Electrical equivalent noise circuit.

the SNR, which includes the sensitivity of the system, is more suitable for AFM applications where displacements are small.

For a given SNR, the MDD of the system is easily calculated by using

$$
\mathrm{MDD}=\frac{1}{\sqrt{\mathrm{SNR}}} .
$$

\section{A. SNR for the optical lever detection method}

In the case of the optical lever detection method, the signal is the difference in the output currents of a split photodetector as shown in Fig. 14 and is given by ${ }^{12}$

$$
i_{s}=\frac{3 \pi a I_{l}}{\lambda l} \xi, \quad I_{l}=\Re \mathcal{R} P,
$$

where $\mathfrak{R}$ and $\mathcal{R}$ is the responsivity of the photodetector and the reflectivity of the cantilever, respectively. The cantilever length is denoted by $l$. The laser power, $P$, is assumed to be incident on a square mirror with dimensions $2 a \times 2 a$ on the cantilever. The signal current is linearly dependent on the cantilever deflection. The sensitivity of the system is defined as current generated by the photodetector per unit displacement of the cantilever and it is given by

$$
S_{l}=\frac{d i_{s}}{d \xi}=\frac{3 \pi a I_{l}}{\lambda l} .
$$

The sensitivity of the lever detection method does not depend on the distance between the cantilever plane and the detector unless the detector is in the near field of the cantilever. The diffraction focal length of the beam is calculated by $(2 a)^{2} / \lambda$, which is typically a few millimeters. After this point, the beam diverges and the change of the laser spot position on the photodetector plane relative to its area remains the same. Hence, placing a photodetector far from the cantilever does not increase the sensitivity of the system. Furthermore, the sensitivity is inversely proportional to the cantilever length. Decreasing the length increases the sensitivity at the expense of increasing the cantilever stiffness.

We next consider the effects of various noise components. To calculate the total mean square shot noise current, the shot noise powers at each photodetector should be added. Hence, the total mean square shot noise current is given by

$$
\left\langle i_{\mathrm{sh}}^{2}\right\rangle=2 q B I_{l} .
$$

Another noise source is the mechanical vibrations of the thermally excited cantilever. The mean square current due to the thermal vibrations of the cantilever is

$$
\left\langle i_{m}^{2}\right\rangle=S_{l}^{2}\left\langle\xi_{n}^{2}\right\rangle=\left(\frac{3 \pi a I_{l}}{\lambda l}\right)^{2}\left\langle\xi_{n}^{2}\right\rangle
$$

where $\left\langle\xi_{n}^{2}\right\rangle$ is the mean square thermal mechanical vibration amplitude of the cantilever.

If the above equations for the signal and noise currents are substituted in Eq. (5.1), the resulting SNR formula for the lever detection method is

$$
\mathrm{SNR}_{\text {lever }}=\frac{S_{l}^{2} /\left\langle i_{\mathrm{sh}}^{2}\right\rangle}{1+S_{l}^{2}\left\langle\xi_{n}^{2}\right\rangle /\left\langle i_{\mathrm{sh}}^{2}\right\rangle+\bar{n}_{\mathrm{ps}}+\left\langle i_{e}^{2}\right\rangle /\left\langle i_{\mathrm{sh}}^{2}\right\rangle} .
$$

This equation is consistent with the equation given in Ref. 4 . In Ref. 4, the SNR formula depends on the cantilever deflection, whereas our formula does not. This is because we de-

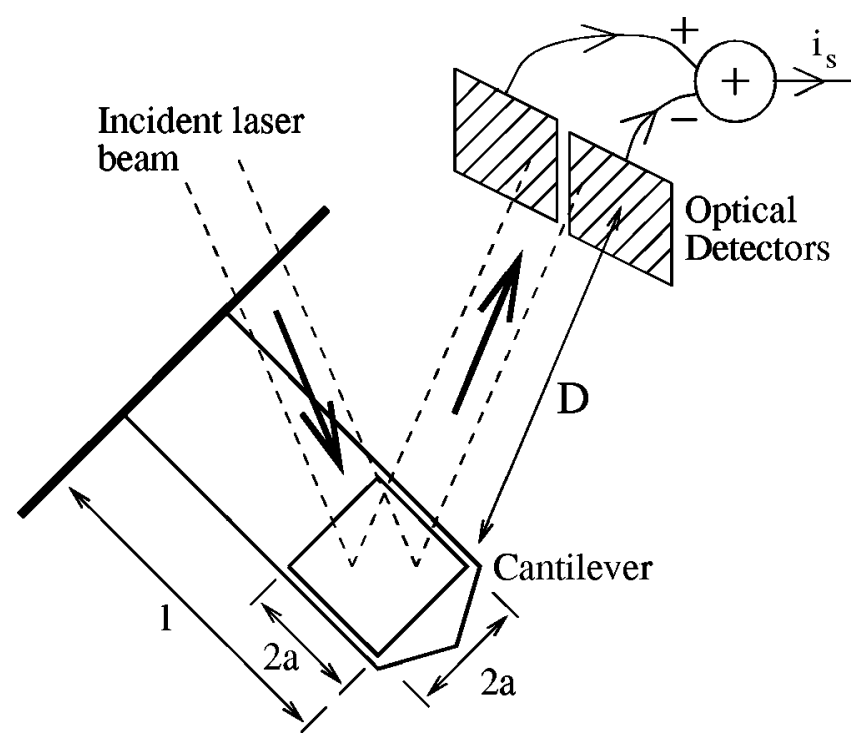

FIG. 14. Optical lever detection method. 
fine the signal to be the derivative of the output current. Moreover, our SNR equation includes other noise sources. The thermal mechanical vibrations of the split photodetector with respect to the cantilever, as well as the vibrations of the laser with respect to the cantilever, contribute to the overall mechanical system noise. $\bar{n}_{\mathrm{ps}}$ contains contributions of the laser pointing noise and the mechanical system noise. The optical lever detection method cannot distinguish the laser pointing noise in the direction normal to the split detector slit from the cantilever motion. The effect of the mechanical system noise is the same as the pointing noise. Hence, both noise components are combined in one variable, $\bar{n}_{\mathrm{ps}}$ (see Appendix B).

\section{B. SNR for interdigital cantilever with one detector}

The deflection of an interdigital cantilever can be determined by measuring either the intensity of the zeroth order component or the first order component. Let us assume that a photodetector is placed at the position of the first order beam and the deflection of the interdigital cantilever is determined by measuring the output current of the detector. By using Eq. (3.3), the detector current can be written as

$$
i_{s}=I_{l} \mathcal{R}_{1} \sin ^{2} \theta,
$$

where $\mathcal{R}_{1}$ shows the ratio of the first order component power to the total power when the moving fingers are deflected by $\lambda / 4$. The sensitivity is given by

$$
S_{\mathrm{ID} 1}=\frac{d i_{s}}{d \xi}=I_{l} \mathcal{R}_{1} \frac{2 \pi}{\lambda} \sin 2 \theta .
$$

The maximum sensitivity is achieved, when the cantilever is deflected by $\lambda / 8$.

If Eq. (5.10) is substituted into the shot noise current formula, the mean square shot noise current is

$$
\left\langle i_{\mathrm{sh}}^{2}\right\rangle=2 q B I_{l} \mathcal{R}_{1} \sin ^{2} \theta .
$$

However, the shot noise depends on the cantilever deflection. The optimum bias should be determined by optimizing the SNR rather than maximizing the sensitivity.

The mechanical noise current is calculated by multiplying the amplitude of the mechanical vibrations of the cantilever by the sensitivity. The SNR formula for the interdigital cantilever with one detector is

$$
\mathrm{SNR}_{\mathrm{ID} 1}=\frac{S_{\mathrm{ID} 1}^{2} /\left\langle i_{\mathrm{sh}}^{2}\right\rangle}{1+S_{\mathrm{ID} 1}^{2}\left\langle\xi_{n}^{2}\right\rangle /\left\langle i_{\mathrm{sh}}^{2}\right\rangle+\bar{n}_{l}+\left\langle i_{e}^{2}\right\rangle /\left\langle i_{\mathrm{sh}}^{2}\right\rangle},
$$

where $\bar{n}_{l}$ is the normalized laser intensity noise, laser phase noise and laser $1 / f$ noise in terms of shot noise (see Appendix B for the definition of the term). Equation (5.13) is nearly same as the SNR formula for the optical lever except that the denominator does not include terms relating to the pointing noise and thermal vibrations of the system. These sources of noise do not contribute to the total noise power in interdigital cantilever system provided that the detector is large enough to collect all power in the first order component. Again we emphasize that we detect intensity rather than position.

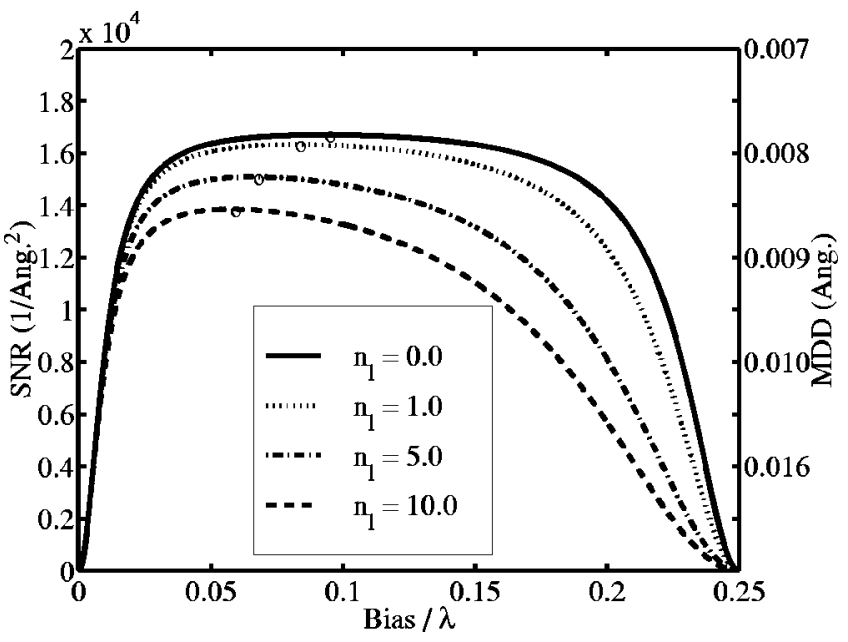

FIG. 15. Calculated SNR and MDD of interdigital cantilever with one detector for various values of $\bar{n}_{l}$. "o", shows optimum bias point. ( $P=1 \mathrm{~mW}$, $\left.\Re=0.54, \mathcal{R}=0.9, \mathcal{R}_{1}=0.185, Q=100, k=1 \mathrm{Nt} / \mathrm{m}, f_{0}=46 \mathrm{kHz}, T=300 \mathrm{~K}\right)$.

For the cantilever used in our experiments, we estimate $\mathcal{R}_{1}$ as 0.185 . This value is calculated by taking the Fourier transform of one-dimensional array of fingers and dividing the power in the first order mode by the total power. Figure 15 plots the SNR and the MDD versus bias point. The optimum bias point does not depend on the amplitude of mechanical noise $\left(\left\langle\xi_{n}\right\rangle\right)$ of the cantilever. It is determined by the value of $\bar{n}_{l}$. As $\bar{n}_{l}$ increases the optimum bias point moves toward zero bias.

We estimate $\bar{n}_{l}$ to be 1 for the ID cantilever with one detector which is a realistic value ${ }^{13}$ if laser intensity, phase and $1 / f$ noise are considered. With this value of $\bar{n}_{l}$, we calculate the SNR of the one detector system as $1.63 \times 10^{4} / \AA^{2}$ at the optimum bias which is around $\lambda / 16$. The corresponding MDD is around $0.0078 \AA$.

\section{SNR for interdigital cantilever with two detectors}

It is also possible to detect the deflection of the interdigital cantilever by using two detectors; one is placed at the position of the first order component and the other at the position of the zeroth order component. In this case, the signal current which is the difference of the output currents of the photodetectors is given by

$$
i_{s}=I_{l}\left(\beta \mathcal{R}_{1} \sin ^{2} \theta-\mathcal{R}_{0} \cos ^{2} \theta\right),
$$

where $\mathcal{R}_{1}$ and $\mathcal{R}_{0}$ show the order intensity relative to the total power at the first order component and at the zeroth order component, respectively. The calculated $\mathcal{R}_{0}$ is 0.23 . $\beta$ is the ratio of the gains of the two channels. The laser intensity noise can be cancelled by choosing $\beta=\mathcal{R}_{0} / \mathcal{R}_{1}$. This assures that signal currents due to the zeroth and the first order components are equal to each other when the cantilever deflection is biased at the optimum value of $\lambda / 8$. The sensitivity of the system is

$$
S_{\mathrm{ID} 2}=2 I_{l} \mathcal{R}_{0} \frac{2 \pi}{\lambda} \sin 2 \theta .
$$




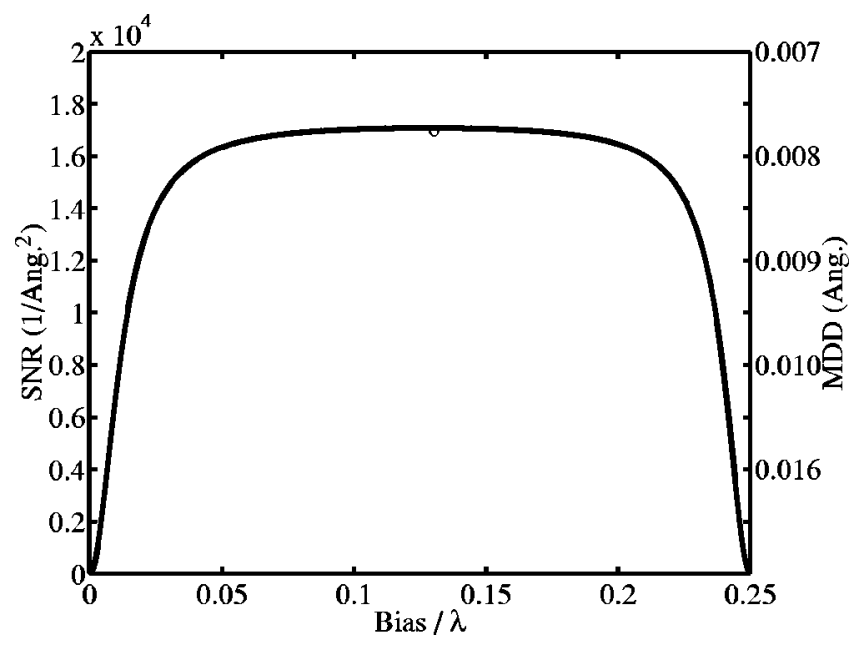

FIG. 16. Calculated SNR and MDD of interdigital cantilever with two detectors. "o" shows optimum bias point $\left(\mathcal{R}_{0}=0.23, \mathcal{R}_{1}=0.185\right)$.

The shot noise power is calculated by adding the noise powers at the outputs of the split photodetector. The mean square shot noise current is

$$
\left\langle i_{\mathrm{sh}}^{2}\right\rangle=2 q B I_{l} \mathcal{R}_{0} .
$$

If a similar derivation is carried out, the SNR of the two detectors system is found to be

$$
\mathrm{SNR}_{\mathrm{ID} 2}=\frac{S_{\mathrm{ID} 2}^{2} /\left\langle i_{\mathrm{sh}}^{2}\right\rangle}{1+S_{\mathrm{ID} 2}^{2}\left\langle\xi_{n}^{2}\right\rangle /\left\langle i_{\mathrm{sh}}^{2}\right\rangle+\left\langle i_{e}^{2}\right\rangle /\left\langle i_{\mathrm{sh}}^{2}\right\rangle} .
$$

The plot of Eq. (5.17) is depicted in Fig. 16. The MDD is $0.0076 \AA$, which is slightly better than interdigital cantilever with one detector. The improvement with respect to the system in Sec. V B is small since the mechanical noise of the cantilever is assumed to be the dominant noise source.

\section{Comparison}

Figure 17 shows the equivalent mechanical noise amplitudes as a function of noise currents for the optical lever, interdigital cantilever with one detector and interdigital can-

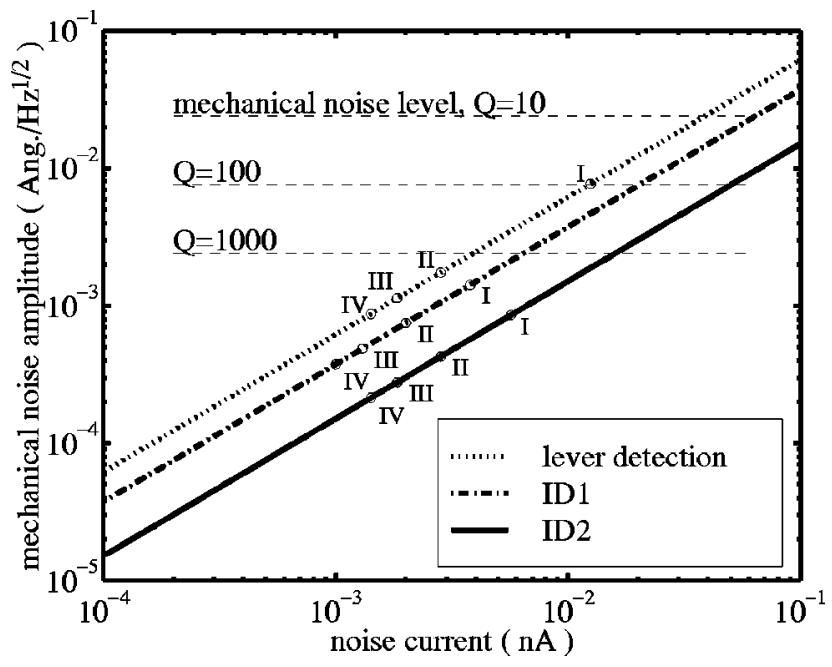

FIG. 17. Equivalent mechanical noise amplitude due to (I) shot noise current, (II) input noise current of op-amp, (III) resistor Johnson noise current, (IV) input noise voltage of op-amp.

tilever with two detectors. The slopes of the lines give the sensitivity for the corresponding system. The most dominant noise source is the thermally excited mechanical vibrations of the cantilevers. The shot noise level is considerably higher for the optical lever. The values of noise currents and equivalent mechanical noise amplitudes are also given in Table I. The total mechanical noise amplitudes gives the MDD of the system. In this table the laser dependent noise sources are neglected. The first three columns show the equivalent output noise currents for each system. The thermal mechanical noises of the cantilevers can be converted to the current by multiplying the mechanical noise amplitudes by the sensitivity of the corresponding system. Since the sensitivity of the third method is the highest, it gives the highest noise current for the same amount of mechanical vibrations. The last three columns show the equivalent noise amplitudes of the cantilever vibrations. These values are calculated by dividing the corresponding currents by sensitivities of the systems.

TABLE I. Calculated square of noise currents and equivalent rms mechanical noise amplitudes due to the various noise sources in different detection methods in $1 \mathrm{kHz}$ bandwidth. Laser dependent noise sources are not considered. $\left(\bar{n}_{l}=0, \bar{n}_{\mathrm{ps}}=0\right)$ input noise current of the amplifier, $\left\langle i_{n}\right\rangle$, is $2 \mathrm{pA} / \mathrm{Hz}^{1 / 2}$. Input noise voltage of the

\begin{tabular}{|c|c|c|c|c|c|c|}
\hline \multirow[b]{2}{*}{$\begin{array}{l}\text { Detection } \\
\text { method }\end{array}$} & \multicolumn{3}{|c|}{ Mean square output noise currents } & \multicolumn{3}{|c|}{ Equivalent mechanical input noise } \\
\hline & $\begin{array}{c}\text { lever } \\
\left(\times 10^{-21} \mathrm{~A}^{2}\right)\end{array}$ & $\begin{array}{c}\mathrm{ID1} \\
\left(\times 10^{-21} \mathrm{~A}^{2}\right)\end{array}$ & $\begin{array}{c}\mathrm{ID} 2 \\
\left(\times 10^{-21} \mathrm{~A}^{2}\right)\end{array}$ & $\begin{array}{l}\text { lever } \\
(\AA)\end{array}$ & $\begin{array}{l}\text { ID1 } \\
(\AA)\end{array}$ & $\begin{array}{l}\text { ID2 } \\
(\AA)\end{array}$ \\
\hline Shot noise & 156.3 & 14.4 & 32.3 & 0.0077 & 0.0014 & 0.0009 \\
\hline $\begin{array}{l}\text { Mechanical noise } \\
(Q=100)\end{array}$ & 152.0 & 410.5 & 2537.5 & 0.0076 & 0.0076 & 0.0076 \\
\hline $\begin{array}{l}\text { Input noise current } \\
\text { of amplifier }\end{array}$ & 8.0 & 4.0 & 8.0 & 0.0017 & 0.00075 & 0.00043 \\
\hline $\begin{array}{l}\text { Input noise voltage } \\
\text { of amplifier }\end{array}$ & 2.0 & 1.0 & 2.0 & 0.00087 & 0.00038 & 0.00021 \\
\hline $\begin{array}{l}\text { Resistor Johnson } \\
\text { noise }\end{array}$ & 3.4 & 1.7 & 3.4 & 0.0011 & 0.00048 & 0.00028 \\
\hline Total noise & 321.7 & 431.6 & 2583.2 & 0.011 & 0.0078 & 0.0077 \\
\hline
\end{tabular}
amplifier, $\left\langle e_{n}\right\rangle$, is $10 \mathrm{nV} / \mathrm{Hz}^{1 / 2}$. The resistor of the transimpedance amplifier is $10 \mathrm{k} \Omega$. 


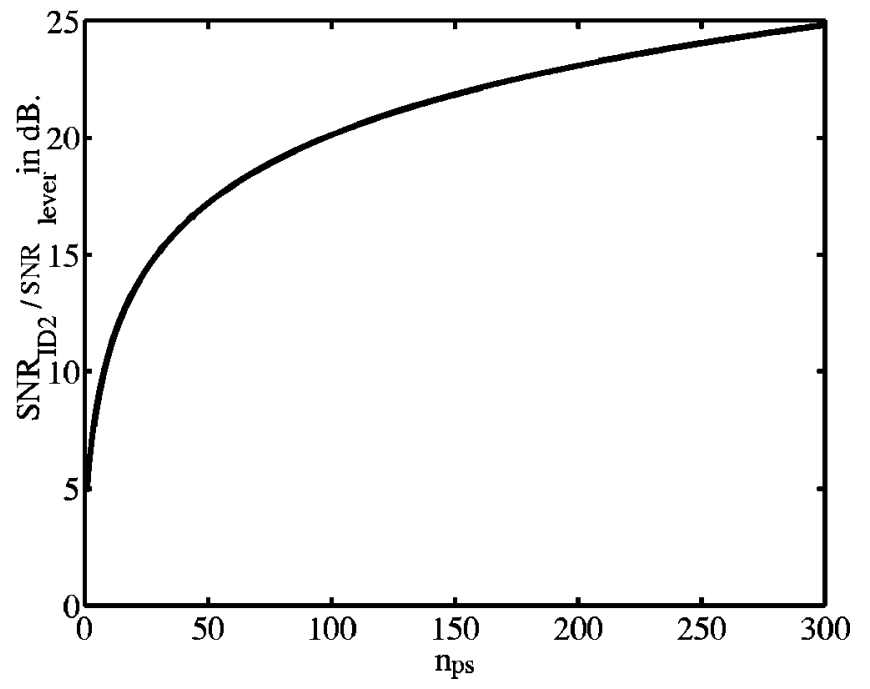

FIG. 18. Calculated SNR ratios of interdigital cantilever with two detectors and lever method. The quality factor of the cantilevers, $Q$, are 100. (For lever detection method: $l=200 \mu \mathrm{m}, a=15 \mu \mathrm{m}$.)

Figure 18 shows the ratio of the SNR of the interdigital cantilever to the optical lever method. When $\bar{n}_{\mathrm{ps}}$ is zero, the interdigital cantilever method is a factor of two better than the optical lever. The interdigital cantilever can achieve the same sensitivity as the optical lever at a lower intensity level.

Theoretically, we find that the MDD of the optical lever is roughly $0.01 \AA$ in a $1 \mathrm{kHz}$ bandwidth if the pointing noise and the mechanical system noise are neglected. The measured MDD is a factor of ten larger than this, which means that the pointing and mechanical noise must be the dominant sources of noise. With an MDD of $0.1 \AA$ of MDD in a $1 \mathrm{kHz}$ bandwidth, we estimate the normalized noise contribution, $\bar{n}_{\mathrm{ps}}$ to be equal to 200 . A primary advantage of the ID can-

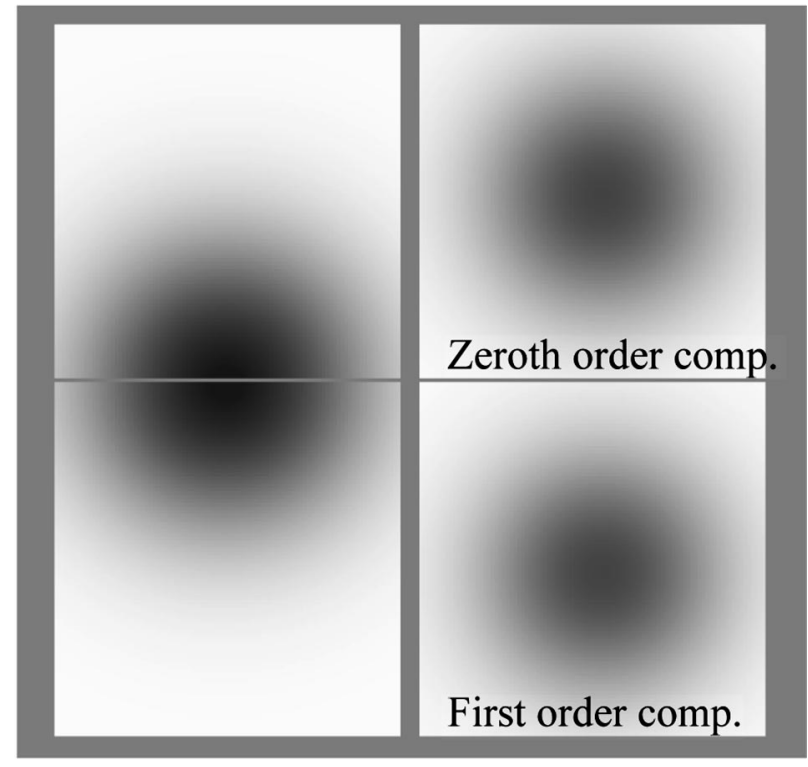

FIG. 19. Positions of the laser spots on the split photodetectors for lever detection method and interdigital cantilever with two detectors. tilever is its immunity from laser pointing noise and mechanical system noise when the detector area is larger than the beam spot size (Fig. 19).

We also note that the ID cantilever does not respond to thermal drifts. The metal layer used to increase the optical reflectivity creates a bimetallic strip which will bend as the temperature changes. However, both arms of the ID cantilever can be designed such that they bend by the same amount. Although the diffracted orders are deflected, the intensity remains constant.

Finally we note that homodyne and heterodyne interferometric deflection detection methods have a sensitivity comparable to the interdigital cantilever. However, the alignment requirements are much more stringent and this makes it difficult to use these sensors for cantilever arrays.

\section{CONCLUSION}

The interdigital cantilever makes use of a microfabricated phase grating to improve the deflection sensitivity and reduce the alignment requirements of the AFM. Operationally, this technique requires an illumination source and a standard photodiode, yet it achieves a resolution that is comparable to the interferometric sensors described previously. The increased simplicity allows the interdigital cantilever to be used in most optical lever AFMs without modification.

In this study, we compare the interdigital cantilever with the optical lever detection method. We conclude that the interdigital cantilever is more sensitive than the optical lever because the interdigital system is insensitive to vibrational movements of the photodetector and laser as well as laser pointing noise. In addition, the interdigital cantilever has the unique property that the sensitivity does not depend on the cantilever length. Since optical intensity is measured, alignment of the photodetector is less crucial than the optical lever where position is measured. We envision that a cylindrically focused laser can be used to illuminate an array of interdigital cantilevers, while a monolithic array of photodiodes is used to image the reflected diffraction pattern in order to determine the deflection of each element.

\section{ACKNOWLEDGMENTS}

This work was supported by Office of Naval Research with partial support from the National Science Foundation.

\section{APPENDIX A: NOISE SOURCES IN OPTICAL AFM SYSTEMS}

In a typical AFM system, there are mainly five sources of noise.

\section{Photodetector noise}

The random arrivals of photons to the photodiode is referred to as shot noise. The mean square shot noise is expressed by $\left\langle\mathbf{i}_{\text {sh }}^{\mathbf{2}}\right\rangle=2 q B I$, where $q$ is the elementary charge, $B$ is the detection bandwidth, and $I$ is the photodiode current. 


\section{Cantilever noise}

All mechanical systems vibrate due to the thermal excitation. The amplitude of acoustical vibration of the thermally excited cantilever is $\left\langle\boldsymbol{\xi}_{\mathbf{n}}^{2}\right\rangle=4 K T B /\left(Q k \omega_{0}\right),{ }^{14,15}$ where $K$ is the Boltzmann constant, $T$ is the temperature, $Q$ is the quality factor of the cantilever, $k$ is the spring constant, and $\omega_{0}$ is the resonance frequency. Corresponding mean square noise current, $\left\langle\mathbf{i}_{\mathbf{m}}^{\mathbf{2}}\right\rangle$, is calculated by multiplying the thermal noise amplitude by the sensitivity of the detection method.

\section{Detection circuit noise}

The noise in the detection circuit is basically due to the Johnson noise, $\left\langle\mathbf{e}_{\mathbf{R}}^{\mathbf{2}}\right\rangle$, of the resistor of the transimpedance amplifier, input noise voltage, $\left\langle\mathbf{e}_{\mathbf{n}}^{2}\right\rangle$, and the input noise current, $\left\langle\mathbf{i}_{\mathbf{n}}^{2}\right\rangle$, of the amplifier and the electrical $1 / f$ noise, $\left\langle\mathbf{i}_{\mathbf{f e}}^{2}\right\rangle$. The mean square Johnson noise is given by $\left\langle e_{R}^{2}\right\rangle$ $=4 K T B R$, where $R$ is the resistor value.

\section{Laser noise}

Laser light is usually obtained from a laser diode which is inherently a noisy device. The main source of the laser noise is the spontaneous emission of photons, resulting in fluctuations in laser intensity and phase. Corresponding noise currents due to the intensity noise and phase noise are denoted by $\left\langle\mathbf{i}_{\text {int }}^{\mathbf{2}}\right\rangle$ and $\left\langle\mathbf{i}_{\text {pha }}^{\mathbf{2}}\right\rangle$. Another noise type in lasers is the pointing noise, which are the random fluctuations of the beam shape and the direction. The mean square pointing noise current is denoted by $\left\langle\mathbf{i}_{\mathbf{p}}^{2}\right\rangle$. Let $\left\langle\theta_{n}^{2}\right\rangle$ show the mean square angular noise amplitude of the laser beam as a result of pointing noise. The corresponding mean square noise current in the lever detection system is given by $\left\langle i_{p}^{2}\right\rangle$ $=\left(S^{2} l^{2} / 9\right)\left\langle\theta_{n}^{2}\right\rangle$, where $S$ is the sensitivity of the system, and $l$ is the length of the cantilever. Finally, lasers exhibit more noise in low frequencies due to the $1 / f$ noise $\left(\left\langle\mathbf{i}_{\mathbf{f l}}^{\mathbf{2}}\right\rangle\right)$.

\section{Overall mechanical system noise}

The overall mechanical parts of the AFM system also vibrate because of the thermal excitation. However, the resonance frequency is low compared to the cantilever. Because of these vibrations the relative position of the laser beam on the detector plane changes. The noise on the laser beam position at the detector plane can be estimated by $\left\langle\xi_{s}^{2}\right\rangle$ $=4 K T B /\left(Q_{s} k_{s} \omega_{0 s}\right)$,, where $Q_{s}, k_{s}$, and $\omega_{0 s}$ are determined by the mechanical properties of the AFM system. Corresponding mean square noise current is given by $\left\langle\mathbf{i}_{\mathbf{m s}}^{2}\right\rangle$ $=\left(S^{2} l^{2} / 9 D^{2}\right)\left\langle\xi_{s}^{2}\right\rangle$, where $D$ is the distance between the cantilever and the photodetector.

\section{APPENDIX B: DEFINITION OF NORMALIZED NOISE QUANTITIES}

In the lever detection method, in order to add the effects of the thermal mechanical system noise and the laser pointing noise, noise currents due to these two noise sources are normalized by the mean square shot noise current. We define a normalized variable, $\bar{n}_{\mathrm{ps}}$, as
TABLE II. Noise currents in different detection methods.

\begin{tabular}{clll}
\hline \hline $\begin{array}{c}\text { Detection } \\
\text { method }\end{array}$ & \multicolumn{1}{c}{$\begin{array}{c}\text { Lever } \\
\text { detection }\end{array}$} & ID1 & ID2 \\
\hline$\left\langle i_{\text {int }}^{2}\right\rangle$ & 0 & n.z. & 0 \\
$\left\langle i_{\text {pha }}^{2}\right\rangle$ & 0 & $\sim 0$ & $\sim 0$ \\
$\left\langle i_{\text {ff }}^{2}\right.$ & 0 & n.z. & 0 \\
$\left\langle i_{p}^{2}\right\rangle$ & n.z. & 0 & 0 \\
$\left\langle i_{e}^{2}\right\rangle$ & n.z. & n.z. & n.z. \\
$\left\langle i_{\text {ms }}^{2}\right\rangle$ & n.z. & 0 & 0 \\
\hline \hline
\end{tabular}

${ }^{a}$ Nonzero.

$$
\bar{n}_{\mathrm{ps}}=\frac{\left\langle i_{p}^{2}\right\rangle+\left\langle i_{\mathrm{ms}}^{2}\right\rangle}{\left\langle i_{\mathrm{sh}}^{2}\right\rangle}
$$

Similarly, the normalized variable, $\bar{n}_{l}$ shows the contributions of the laser intensity noise, the laser phase noise and the laser $1 / f$ noise to the interdigital system with one detector in terms of the mean square shot noise,

$$
\bar{n}_{l}=\frac{\left\langle i_{\text {int }}^{2}\right\rangle+\left\langle i_{\text {pha }}^{2}\right\rangle+\left\langle i_{\mathrm{fl}}^{2}\right\rangle}{\left\langle i_{\mathrm{sh}}^{2}\right\rangle} .
$$

Note that the shot noise levels are different for both detection systems. For the interdigital cantilever with one detector, the shot noise level is given by

$$
\left\langle i_{\mathrm{sh}}^{2}\right\rangle=q B \mathcal{R}_{1} I_{l},
$$

when the cantilever is biased to $\lambda / 8$.

Table II summarizes the contributions of various noise sources. Detection systems that use split photodetectors are insensitive to the laser intensity noise. Laser phase noise does not contribute to the total noise power in the lever detection method. Phase noise is usually converted to the amplitude noise in interferometric systems. However, the amplitude of the noise depends on the optical path difference between each arm of the interferometer. In the interdigital cantilever system, the optical path is nearly same for specularly reflected light and diffracted light. Hence, phase noise is very small.

${ }^{1}$ G. Binnig, C. F. Quate, and Ch. Gerber, Phys. Rev. Lett. 56, 930 (1986).

${ }^{2}$ Y. Martin, C. C. Williams, and H. K. Wickramasinghe, J. Appl. Phys. 61, 4723 (1987).

${ }^{3}$ D. Rugar, H. J. Mamin, and P. Guethner, Appl. Phys. Lett. 55, 2588 (1989).

${ }^{4}$ G. Meyer and N. M. Amer, Appl. Phys. Lett. 53, 1045 (1988)

${ }^{5}$ S. Alexander, L. Hellemas, O. Marti, J. Schneir, V. Elings, P. K. Hansma, M. Longmire, and J. Gurley, J. Appl. Phys. 65, 164 (1989).

${ }^{6}$ M. Tortonese, R. C. Barrett, and C. F. Quate, Appl. Phys. Lett. 62, 834 (1993).

${ }^{7}$ S. C. Minne, S. R. Manalis, and C. F. Quate, Appl. Phys. Lett. 67, 3918 (1995).

${ }^{8}$ S. R. Manalis, S. C. Minne, A. Atalar, and C. F. Quate, Appl. Phys. Lett. 69, 3944 (1996).

${ }^{9}$ ANSYS, Inc., 201 Johnson Road, Houston, PA 15342-0065.

${ }^{10} \mathrm{~J}$. W. Goodman, Introduction to Fourier Optics (McGraw-Hill, New York, 1968)

${ }^{11}$ K. Iizuka, Engineering Optics (Springer, New York, 1986). 
${ }^{12}$ D. Sarid, Scanning Force Microscopy (Oxford University Press, New York, 1991).

${ }^{13}$ K. Petermann, Laser Diode Modulation and Noises (Kluwer Academic, Dordrecht, 1988).
${ }^{14}$ U. Durig, J. K. Gimzewski, D W. Pohl, and R. Schlittler, Phys. Rev. Lett. 57, 2403 (1986)

${ }^{15}$ G. M. McClelland, R. Erlandson, and S. Chang, Review of Progress in Quantitative Non-Destructive Evaluation (Plenum, New York, 1987). 